\title{
Erratum
}

\section{On the structure of complete Kähler manifolds with nonnegative curvature near infinity}

\section{Peter Li}

Department of Mathematics, University of Arizona, Tucson, AZ 85721, USA

Invent. Math. 99, 579-600 (1990)

We would like to thank Harold Donnelly for pointing out that in the conclusion of Theorem 3.3, the manifold $M$ might not necessarily split into Riemannian product of $N$ with a Riemann surface $\Sigma / D_{0}$. The correct conclusion is that, for each end $e$ of $M$, it is the total space of a holomorphic fibration over a complete Riemann surface with boundary $\Sigma$ with totally geodesic fibres given by $N$. The Riemann surface is homeomorphic to a half cylinder $\mathbf{S}^{1} \times \mathbf{R}^{+}$and has nonnegative Gaussian curvature. The fibre $N$ is a compact Kähler manifold with nonnegative sectional curvature. Moreover, the metric of $M$ is locally given by the product metric of $\Sigma \times N$. To clarify this point, let us elaborate on the argument.

On page 590, paragraph 3 , we have shown that at each end, the level sets of the holomorphic function $h$ must be totally geodesic at the regular values of $H$. Since the limit of totally geodesic submanifolds is itself a smooth totally geodesic submanifold, we conclude that all the level sets are in fact totally geodesic submanifolds of $M$. We claim that locally, the metric splits into a product metric. To see this, let us pick an orthonormal frame field $\left\{e_{1}, \ldots, e_{2 m}\right\}$ of $M$ which has the property that $\left\{e_{1}, \ldots, e_{2 m-2}\right\}$ are tangent to the level sets of $h, e_{2 m-1}=\nabla \beta$, and $e_{m}=J \nabla \beta$. Since the Busemann function $\beta$ satisfies $|\nabla \beta| \equiv 1$ and its integral curves are geodesics, we see that $\nabla_{e_{2 m-1}} e_{2 m-1}=0$. To establish the splitting locally, it suffices to show that the involutive distribution $T$ spanned by $\left\{e_{1}, \ldots, e_{2 m-2}\right\}$ is parallel. This is equivalent to proving that $\left\langle\nabla_{X} e_{i}, e_{v}\right\rangle=0$ for all vector $X$ tangent to $M$, for all $1 \leqq i \leqq 2 m-2$, and for all $2 m-1 \leqq v \leqq 2 m$. If $X=e_{j}$ for some $1 \leqq j \leqq 2 m-2$, this is clearly valid due to the fact that the level sets of $h$ are totally geodesic submanifolds. The term

$$
\begin{aligned}
\left\langle\nabla_{e_{2 m-1}} e_{i}, e_{2 m-1}\right\rangle & =-\left\langle\nabla_{e_{2 m-1}} e_{2 m-1}, e_{i}\right\rangle \\
& =0
\end{aligned}
$$

for all $1 \leqq i \leqq 2 m-2$, by the fact that $e_{2 m-1}=\nabla \beta$. The Kähler condition implies that

$$
\begin{aligned}
\left\langle\nabla_{e_{2 m-1}} e_{i}, e_{2 m}\right\rangle & =\left\langle\nabla_{e_{2 m-1}} e_{i}, J e_{2 m-1}\right\rangle \\
& =-\left\langle\nabla_{e_{2 m-1}} J e_{i}, e_{2 m-1}\right\rangle \\
& =0 .
\end{aligned}
$$


Observe that since $T$ is the distribution given by the tangent spaces of the level set of a holomorphic function, its orthogonal complement is also a involutive distribution spanned by $\left\{e_{2 m-1}, e_{2 m}\right\}$. Hence,

$$
\begin{aligned}
\left\langle\nabla_{e_{2 m}} e_{i}, e_{2 m-1}\right\rangle & =-\left\langle\nabla_{e_{2 m}} e_{2 m-1}, e_{i}\right\rangle \\
& =-\left\langle\nabla_{e_{2 m-1}} e_{2 m}, e_{i}\right\rangle \\
& =0 .
\end{aligned}
$$

Similarly, we conclude that

$$
\begin{aligned}
\left\langle\nabla_{e_{2 m}} e_{i}, e_{2 m}\right\rangle & =-\left\langle\nabla_{e_{2 m}} J e_{i}, e_{2 m-1}\right\rangle \\
& =0 .
\end{aligned}
$$

This implies that the metric must locally split into a product metric. In particular, the totally geodesic fibres are all isometric. If the end is simply connected, de Rham decomposition theorem implies that it must be globally a product of $N$ with a Riemannian surface. However, in general, the global splitting only occurs on the universal covering. We would also like to point out that if the level sets of regular values of $h$ have more than one component, when the value approaches a singular value, even though the limiting level set is a totally geodesic submanifold, it could however have multiplicity more than 1 . This is indicated by the simple example given by the map $z \mapsto z^{k}$. Let us now denote the connected totally geodesic submanifolds by $N$. Clearly $N$ is given by the connected components of level sets of $h$.

It was established in [L-T1] that the end $e$ is homeomorphic to the product $(\partial C(2) \cap e) \times[0, \infty)$, where $\partial C(2)$ is the level of the Busemann function at value 2. Moreover, if we denote $S(t)=\partial C(t) \cap e$, then $S(t)$ is homeomorphic to $S=S(2)$. The universal covering $\tilde{e}$ of $e$ is clearly homeomorphic to $\tilde{S} \times[0, \infty)$, where $\widetilde{S}$ is the universal covering of $S$, and $\pi_{1}(e)$ acts on the preimage $\widetilde{S}(t)$ of the level sets $S(t)$. Let $\widetilde{P}(a)$ be the preimage of the level set $P(a)$ of $h$, then the preimage $\tilde{N}$ of $N$ in $\widetilde{P}(a)$ are totally geodesic submanifolds of $\tilde{e}$ and are contained in some $\tilde{S}(t)$. The global isometric and holomorphic splitting in $\tilde{e}$ is then of the form $\widetilde{N}_{0} \times \tilde{\Sigma}$ where $\widetilde{N}_{0}$ is a connected component of $\tilde{N}$ and $\tilde{\Sigma}$ is some Riemann surface with boundary. Since the Busemann function is constant on $N$, it can be lifted to defined on $\tilde{\Sigma}$. Hence $\Sigma$ must be homeomorphic to $\mathbf{R} \times[0, \infty)$, and each $\tilde{S}(t)$ is isometrically $\tilde{N} \times \mathbf{R}$. Note that the metric of $\mathbf{R}$ might not be smooth and might vary for different values of $t$. An element of $\pi_{1}(e)$ must be of the form $(f, g)$ where $f$ is an isometry of $\tilde{N}$ and $g$ is an isometry of $\mathbf{R}$. Since $M$ is a complex manifold, $N$ is a complex submanifold, and the action of $\pi_{1}(e)$ on $S$ is oriented, the isometry $g$ must be orientation preserving. This implies that $g$ must be a translation. In particular, the set of isometries of $\mathbf{R}$ which induced from $\pi_{1}(e)$ must be a group generated by some translation $g_{0}$ of $\mathbf{R}$. The Riemann surface obtained by $\Sigma=\widetilde{\Sigma} /\left\langle g_{0}\right\rangle$ is homeomorphic to a half cylinder. Moreover, if we denote the isometry group $F$ of $\tilde{N}_{0}$ given by the set of isometries $f$ which occurs as $(f, i d) \in \pi_{1}(e)$, then $e$ is clearly the total space of the fibration over $\Sigma$ with totally geodesic fibers given by $N=N_{0} / F$. By the assumption on the curvature of $M$, it is clear that $\widetilde{\Sigma}$ has nonnegative Gaussian curvature. In particular, $\Sigma$ has nonnegative Gaussian curvature.

We would like to point out that the fibration might not be a Riemannian fibration. When the fibration happens to be a Riemannian fibration, it still might not be trivial. This was pointed out by Donnelly by considering a nontrivial $\mathbf{S}^{2}$ fibre bundle over the flat cylinder. 